\title{
Step-up Therapy with Skin Barrier Repair Emulsion in Personal Protective Equipment (PPE) Associated Adverse Skin Reactions during the COVID-19 Pandemic
}

Katlyn Anderson, PA-C Arkansas Dermatology and Skin Cancer Center, Little Rock, AR

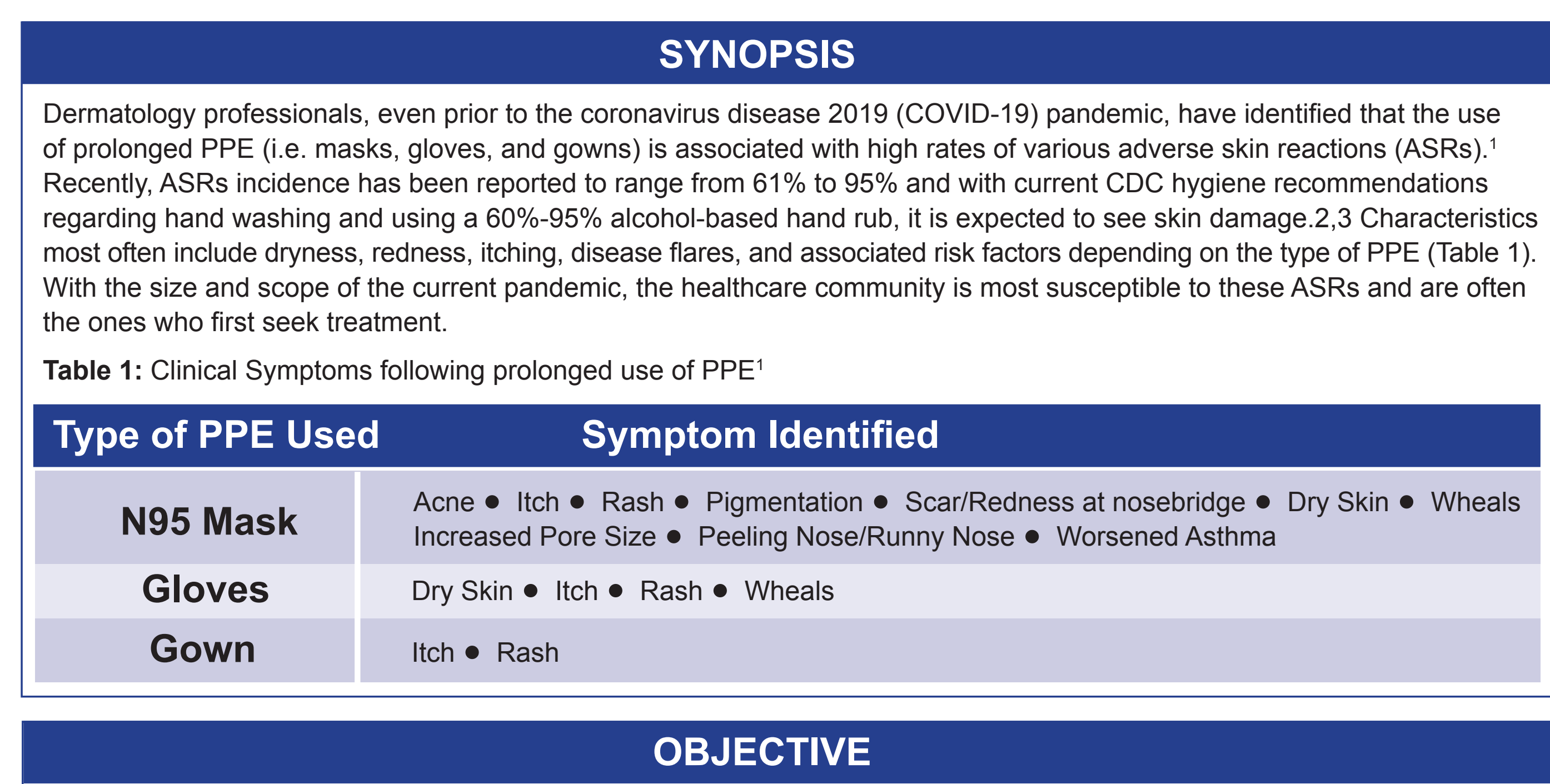

EpiCeram is a skin barrier repair emulsion containing ceramides, conjugated linoleic acid, and cholesterol (as a 3:1:1 ratio) in an emollient base. Lipid-based barrier repair therapy, if comprised of the 3 key stratum corneum lipids, in sufficient quantitit variety of dermatoses. ${ }^{4}$

Vhe ain was lo ideriny indudual cases of PPE assoch

The aim was to identify individual cases of PPE associated ASRs in healthcare workers and evaluate the step-up therapy and maintenance use of a lipid based barrier repair therapy.

\section{CONCLUSIONS}

Step-up and maintenance therapy with a 3:1:1 skin barrier repair emulsion was associated with improved outcomes in PPE associated skin irritation on the hands. As COVID-19 has enhanced a focus on proper hygiene and PPE, ASRs are likely to become more prevalent not only in the healthcare community but at some point will move towards frontline workers, in general. It is therefore important to re-evaluate and evolve approaches in the prevention, treatment, and maintenance of PPE associated ASRs. Further well controlled analyses are needed to elucidate the findings of this case report.

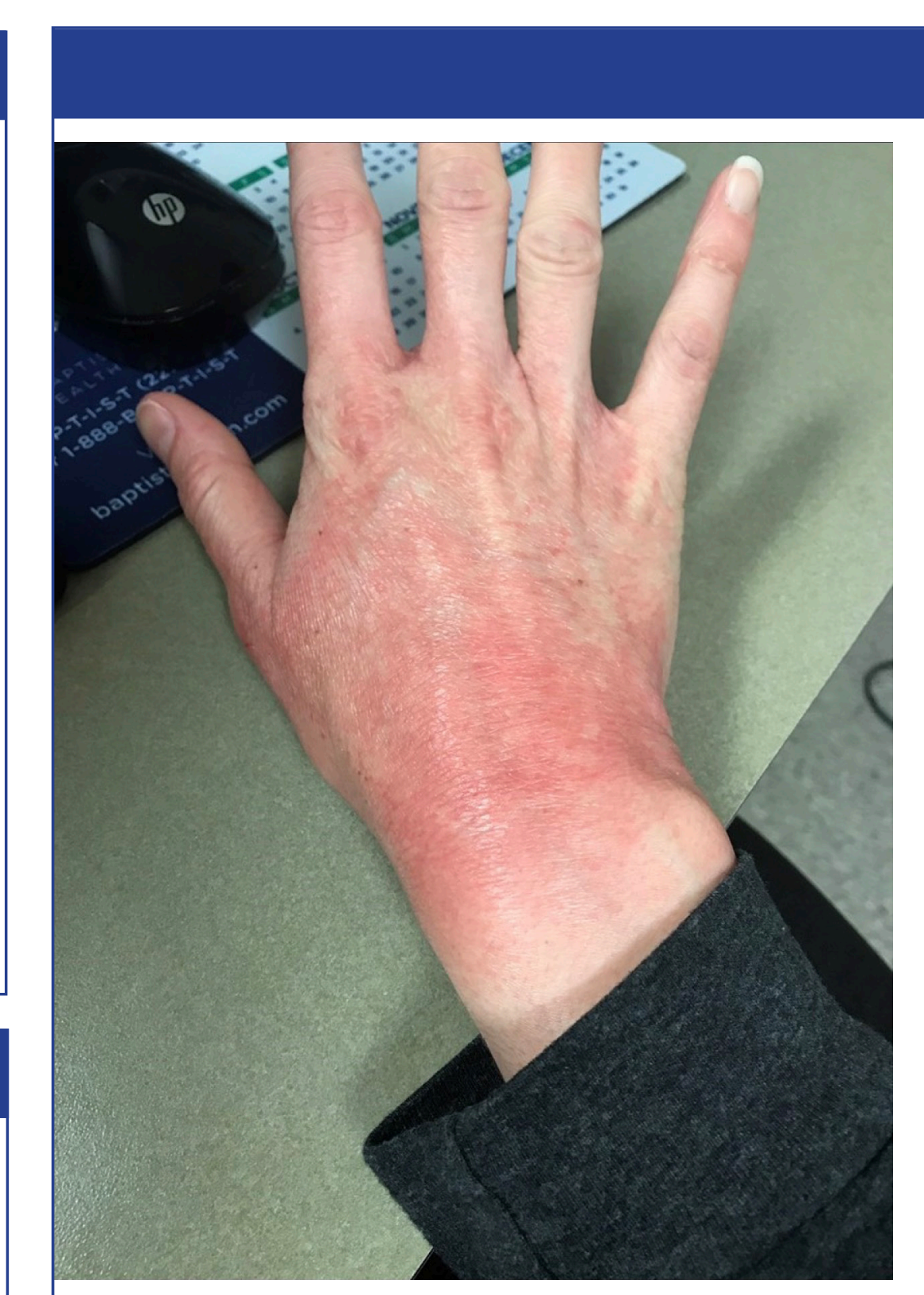

PRESENTATION: 30-year-old female nurse noticed a hand rash following PPE attire (repeated gloves) The rash became erythematous itchy failed treatments included: variety of over-thecounter ointment and creams utilized for weeks, mometasone and tacrolimus - all of which provided no relief.

METHODS
This patient case report reviews the signs,
symptoms, diagnostic work-up, treatment, and
follow up of a a 0-year old female nurse working
in Allergy and Asthma.
DISCLOSURES
KISC
Katlyn Anderson is a paid consultant to
Primus Pharmaceuticals.

RESULTS

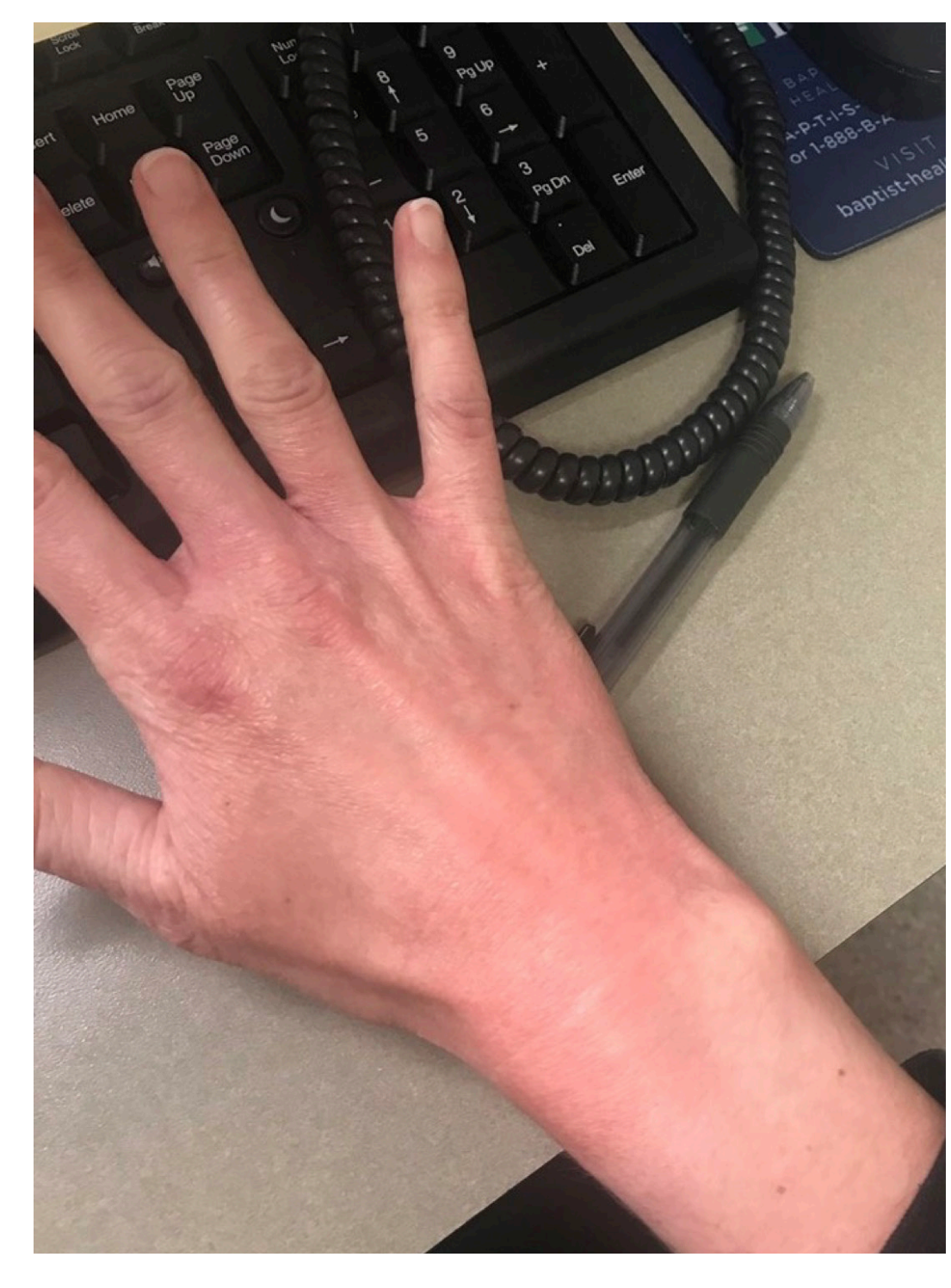

2-WEEKS: The patient was given clobetasol for weeks once daily and EpiCeram, a skin barrie repair emulsion containing ceramides, conjugated linoleic acid, and cholesterol (as a 3:1:1 ratio) in an emollient base, once to twice daily as a stepup therapy. The patient stated improvement in pain, blistering, and fissuring within 2 to 3 days of use. A few days later,
also began to improve.

\begin{tabular}{|c|}
\hline REFERENCES \\
\hline 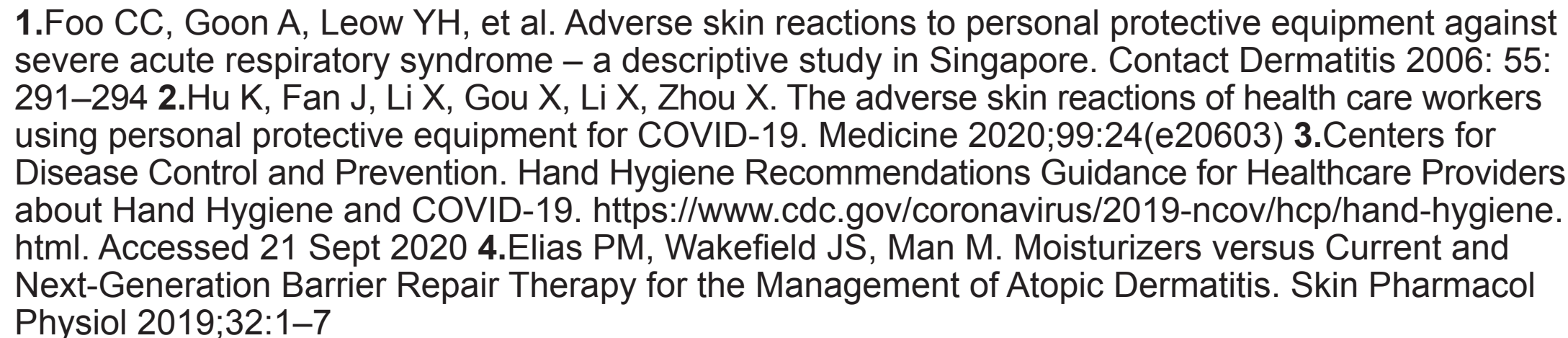 \\
\hline
\end{tabular}

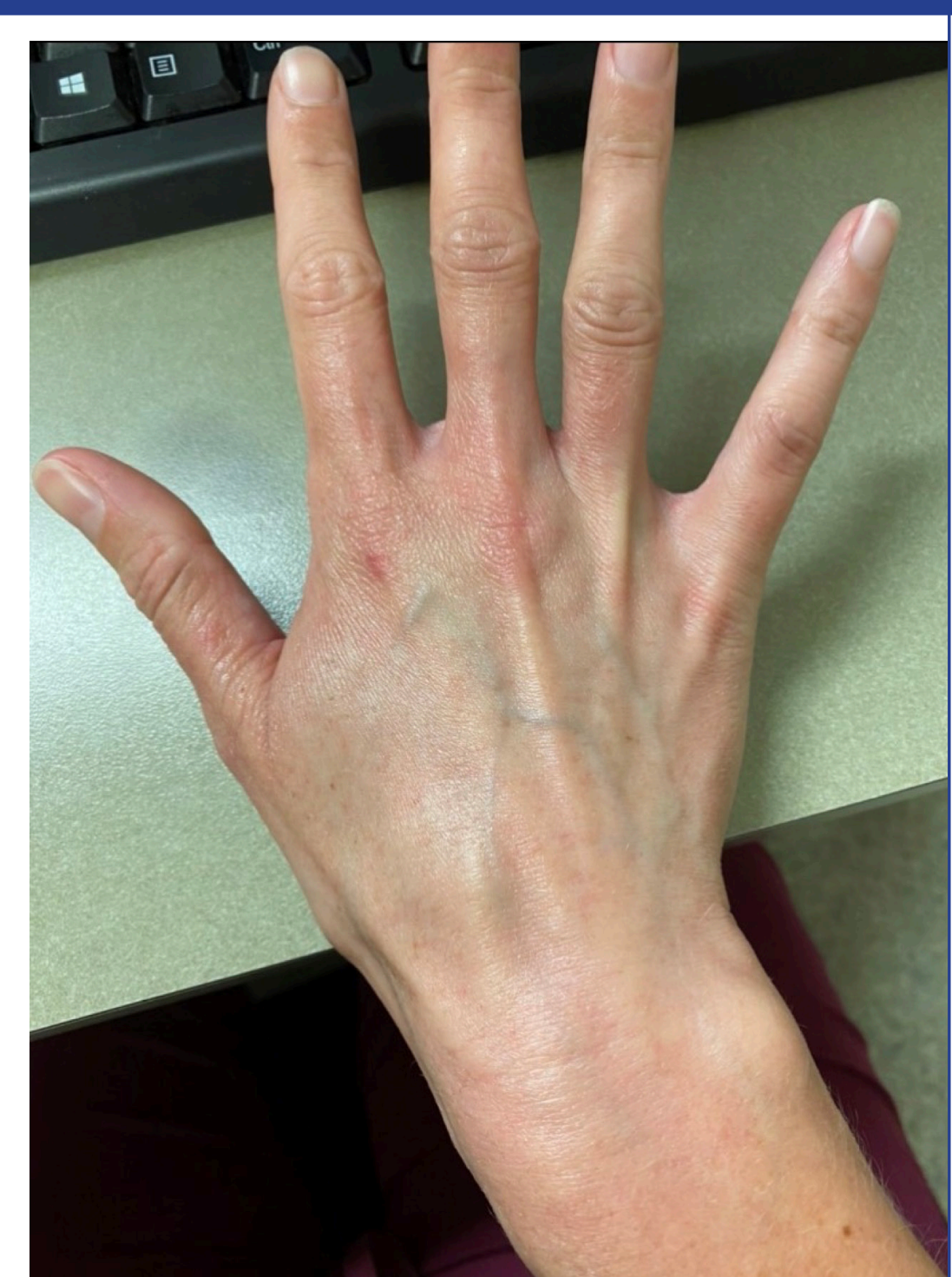

MAINTENANCE PHASE (6MONTHS): The patient continues to use skin barrier repair emulsion daily as a preventative measure after associated skin iritation on her hands and has corporated lifestyle changes (i.e. chemical free soaps and detergents). each hand wash and reports no further PPE 